\title{
Atlas of picocyanobacteria monoclonal strains from the collection of CNR-IRSA, Italy
}

\author{
Cristiana Callieri, ${ }^{1 *}$ Edoardo Mandolini,${ }^{1,2}$ Roberto Bertoni, ${ }^{1}$ Rosaria Lauceri, ${ }^{1}$ Antonio Picazo, ${ }^{3}$ Antonio Camacho, ${ }^{3}$ \\ Pedro J. Cabello-Yeves ${ }^{4}$
}

${ }^{1}$ National Research Council (CNR), Institute of Water Research (IRSA), Verbania, Italy; ${ }^{2}$ Department of Microbiology, University of Innsbruck, Innsbruck, Austria; ${ }^{3}$ Cavanilles Institute of Biodiversity and Evolutionary Biology, University of Valencia, Spain;

${ }^{4}$ Evolutionary Genomics Group, Departamento de Producción Vegetal y Microbiología, Universidad Miguel Hernández, San Juan de Alicante, Alicante, Spain

\begin{abstract}
This atlas aims to provide an overview of the picocyanobacteria cultures hold by the CNR-IRSA collection. These are monoclonal strains mostly of the genera Synechococcus and Cyanobium. These strains were mainly isolated from lakes of different trophic status around the globe and, so far, they are not available in other collections of algae and (pico)cyanobacteria. Here, we summarise the main characteristics of these strains, including some previously published data, and offer most of the strains as material available for research to every scientist upon request.
\end{abstract}

\section{INTRODUCTION}

Picocyanobacteria are tiny autotrophic cells appearing in nature both as coccoid- or rod-shaped single cells, with the minor axis shorter than $2 \mu \mathrm{m}$, as well as microcolonies (Callieri et al., 2012). The most widespread genera are Synechococcus and Cyanobium, both belonging to the phylum of Cyanobacteria, which include some of the oldest microorganisms that played an important role to the oxygenation of the oceans and, ultimately, of Earth (Hamilton et al. 2016; Sanchez-Baracaldo et al., 2019). The Synechococcus spp. are widely distributed throughout aquatic systems of the world, greatly contributing to the global primary production (Flombaum et al., 2013). Synechococcus cell wall is of Gram-negative type, but showing a thick peptidoglycan layer that makes the organisms particularly robust to mechanical stress. In the cytoplasm, these cells show phosphate inclusions, glycogen granules, and highly structured carboxysomes which host

Corresponding author: cristiana.callieri@irsa.cnr.it

Key words: Picocyanobacteria collection; Synechococcus spp.; strain isolation, monoclonal cultures.

Edited by: Diego Fontaneto, CNR-IRSA, Verbania, Italy

Received: 9 January 2021.

Accepted: 1 March 2021.

This work is licensed under a Creative Commons Attribution NonCommercial 4.0 License (CC BY-NC 4.0).

${ }^{\circ}$ Copyright: the Author(s), 2021

Licensee PAGEPress, Italy

J. Limnol., 2021; 80(1):2002

DOI: 10.4081/jlimnol.2021.2002 the enzyme ribulose-1,5-bisphosphate carboxylase/ oxygenase ( $\mathrm{RuBis} \mathrm{CO})$, involved in the carbon fixation process. Furthermore, coupled to the machinery for photosynthesis, Synechococcus cells have a sophisticated light-harvesting system called phycobilisome (PBS). The PBS has a complex structure with an allophycocyanin (AP) core surrounded by rods made by other phycobiliproteins, specifically phycocyanin $(\mathrm{PC})$ and phycoerythrin $(\mathrm{PE})$. This pigment diversity allows Synechococcus to colonize a wide range of light niches in planktonic habitats (Stomp et al., 2007; Six et al., 2007; Farrant et al., 2016).

Molecular analyses on Synechococcus genomes (Dufresne et al., 2008; Scanlan et al., 2009) have revealed the presence of genes for the synthesis and regulation of many processes, further emphasizing the critical role of Synechococcus spp. in aquatic systems (Scanlan 2012; Callieri et al., 2012). New studies on the genome of nonmarine Synechococcus recently published (Cabello-Yeves et al., 2017; Di Cesare et al., 2018, Cabello-Yeves et al., 2018, Sanchez-Baracaldo et al., 2019) emphasized the need to provide more insights on the relationships among the different clades of Synechococcus spp. derived from freshwater, brackish and marine environments.

The analysis of $16 \mathrm{~S}$ rRNA from marine Synechococcus strains showed the presence of three sub clusters containing strains of marine origin, 5.1, 5.2, and 5.3, as well as 13 nonmarine clusters (Fuller et al., 2003; Callieri et al., 2013). Whereas cluster 5.1 only includes marine strains, clusters 5.2 and 5.3 include marine, brackish and freshwater strains. To fully appreciate the phylogenetic relationships among all the clusters, it is of paramount importance to isolate and sequence as many new strains as possible from both freshwater and brackish environments, whose investigations are still scarce to date. Altogether, more detailed genomic, ecological, and evolutionary studies would raise awareness on the essential role that these organisms play in the biogeochemical cycles of aquatic ecosystems. Thus, in this 
atlas, we present 25 monoclonal strains of picocyanobacteria currently cultured at the CNR-IRSA (Italy) cell collection. These strains, coming from a great variety of habitats of different geographical locations, are physiologically characterized. The strains have been isolated from freshwater and brackish environments, including three strains isolated from samples of the Black Sea obtained from the depths of 6.5 and $750 \mathrm{~m}$ (Callieri et al., 2019). Some of the strains presented have their genome already sequenced and almost all have the $16 \mathrm{~S}$ rRNA sequenced. The atlas provides data on the strains, from pictures made by epifluorescence microscopy to flow cytometry characterization, giving information of their size, volume, and absorbance spectra.

We kindly underline that most of the Synechococcus strains or other genera (Cyanobium, Vulcanococcus) reported in this atlas can be requested for scientific purposes to the corresponding author.

\section{DATA DESCRIPTION}

The twenty-five picocyanobacteria strains were described using epifluorescence microscopy, spectrophotometry, spectrofluorometry, flow cytometry, and genomic analyses. Detailed characteristics of the strains, together with their original location, depth of sampling, genome and 16S rRNA sequence accession numbers, are reported in Tab. 1.

The analysis under the epifluorescence microscope showed that the picocyanobacteria have a simple cell morphology, typically tending to either the coccoid or the bacillary form with an average cell volume of $0.5 \mu \mathrm{m}^{3}$, comprised between strain Morenito 9A2 having the smallest average cell volume $\left(0.06 \pm 0.08 \mu \mathrm{m}^{3}\right)$ and strain La Cruz $8 \mathrm{H} 5$ with the largest one $\left(2.28 \pm 0.48 \mu \mathrm{m}^{3}\right)$. The general dimension trend is of small cells, mainly of coccoid shape, with only strains BO8801, MW73D5, ATX 6E5, Cyanobium Tous-M-B4, BS55D and BSA11S displaying bacillar form. The difference in shape and size could be observed well at the microscopy images taken for each organism by the epifluorescence microscope camera. In some of these pictures, it was also possible to detect some cell clusters (e.g., Cyanobium usitatum, AMD-g), suggesting the capacity to form microcolonies.

The pigment composition of the strains is indicated from the absorbance spectra measured by spectrophotometric analysis, allowing a first clear separation of cells in PE cell and PC cell strains. In all absorption spectra measured, we observed absorption peaks corresponding to the main cyanobacterial pigments: chlorophyll- $a$ (one in vivo peak at $440 \mathrm{~nm}$ and a second one at $680 \mathrm{~nm}$ ), carotenoids (peak at $500 \mathrm{~nm}$ ), phycoerythrin (peak at 570/580 nm), phycocyanin and allophycocyanin (peaks at $620 \mathrm{~nm}$ and $650 \mathrm{~nm}$, respectively). According to the fluorescence emitted by the two representative phycobiliproteins, phycoerythrin and phycocyanin, the substantial differences between the two cell types (PE and PC cells) was described (Wood et al., 1985; Ong and Glazer, 1991). UV-Vis absorption spectra of PE cells and PC cells are characterized by the presence or absence, respectively, of the absorbance peak between 550$570 \mathrm{~nm}$, typical of phycoerythrin (Callieri et al., 1996). PC cells have a prevalence of phycocyanin, showing a peak between 610 and $640 \mathrm{~nm}$, less visible in the PE cells, but still detectable. In most of PE cell's absorbance spectra (e.g., Vulcanococcus limneticus LL) five absorption peaks of the pigments could be sequentially observed: chlorophyll- $a$, carotenoids, phycoerythrin, phycocyanin and, again, chlorophyll- $a$. Contrastingly, PC cells lack the phycoerythrin peak in their absorption spectra (e.g., Morenito 9A2).

$\mathrm{PE}$ and PC picocyanobacteria types have specific cytograms and show a dominant cytometric cloud according to their pigments. The cytograms obtained at FL2-H (phycoerythrin) vs FL3-H (chlorophyll-a), confirm the existence of two distinct footprints, depending on the pigment composition of the cells. Flow cytometer analyses, in particular the FL2-H vs FL3-H cytograms, allowed for the characterization of the strains, depending on their pigment type (PE or PC) and based on the intensity of the autofluorescence signal detected. The clouds of density with fluorescence values of FL2-H between $10^{3}$ and $10^{5}$ and FL3$\mathrm{H}$ between $10^{3}$ and $10^{6}$ corresponded to events displaying prominent phycoerythrin fluorescence. Clouds with fluorescence values of FL2-H between 10 and $10^{3}$ and FL3$\mathrm{H}$ between $10^{3}$ and $10^{6}$ corresponded to events displaying dominant phycocyanin/allophycocyanin fluorescence. Events recorded with FL3-H below $10^{3}$ were generally considered debris or dead cells (Olson et al., 1989).

Spectrofluorimetric analysis was performed for a more precise characterization of the accessory pigments of the strains on three representative picocyanobacteria, BO8801 (PC), Nahuel Huapi 1 G10 (PE) and Aljojuca 7D2 (PE), by recording their emission and excitation spectra (Fig. 1). Emission spectra were obtained exciting at $436 \mathrm{~nm}$ (chlorophyll- $a$ absorbance peak) and at $520 \mathrm{~nm}$ (at a shorter wavelength than the phycoerythrin absorbance peak). In the first case $\left(\lambda_{\mathrm{ex}}=436 \mathrm{~nm}\right)$ (Fig. 1B), the emission of chlorophyll- $a$ was visible (around $680 \mathrm{~nm}$ ), as well as the emission of the main accessory pigment: phycoerythrin (around $580 \mathrm{~nm}$ ) for PE cell strains (N.Huapi 1G10 and Aljojuca 7D2) and phycocyanin (around $650 \mathrm{~nm}$ ) for the PC cell strain (BO8801). When the excitation wavelength was set at $520 \mathrm{~nm}$ (outside the chlorophyll- $a$ absorbance peak and nearer the phycoerythrin absorbance peak) (Fig. 1A), chlorophyll- $a$ emission was greatly reduced and was no more detectable in the BO8801 strain, while phycoerythrin emission peak $(580 \mathrm{~nm})$ was predominant in the spectra of N.Huapi 1 G10 and Aljojuca 7D2 strains. This peak was absent in the BO8801 strain, confirming that phycoerythrin was not produced by this organism. Excitation spectra were executed with $\lambda_{\text {em }}$ at $680 \mathrm{~nm}$ (chlorophyll- $a$ peak) and 580 $\mathrm{nm}$ (phycoerythrin peak). The contribution to the emission 
at $680 \mathrm{~nm}$ was monitored from $400 \mathrm{~nm}$ to $670 \mathrm{~nm}$ (Fig. 1C), whereas the contribution to the emission at $580 \mathrm{~nm}$ was monitored from $450 \mathrm{~nm}$ to $570 \mathrm{~nm}$ (Fig. 1D). In both cases, the excitation spectra showed the presence of phycoerythrin as main accessory pigment for N.Huapi $1 \mathrm{G} 10$ and Aljojuca
7D2 strains (580 nm), and of phycocyanin as main accessory pigment for BO8801 strain (between 610 and 640 $\mathrm{nm})$. However, the shape of the excitation spectra was quite different from that of the absorbance spectra as a consequence of the energy transfer processes that transport

Tab. 1. List of 25 picocyanobacterial monoclonal strains isolated from different aquatic systems. From left: general information on the strain, dimensions and accession number for genome sequence (Sanchez Baracaldo et al., 2018) or for 16SrRNA (Callieri et al., 2013), epifluorescence microscopy image (1250x, mark $2 \mu \mathrm{m}$; PE: blue filter-set and PC: green filter-set), specific absorbance spectra (red, phycoerythrin (PE); green, phycocyanin (PC)) and cytogram (FL2 vs FL3). For the strains whose complete genome is available, the accession number to the Genome OnLine Database (GOLD) is included.

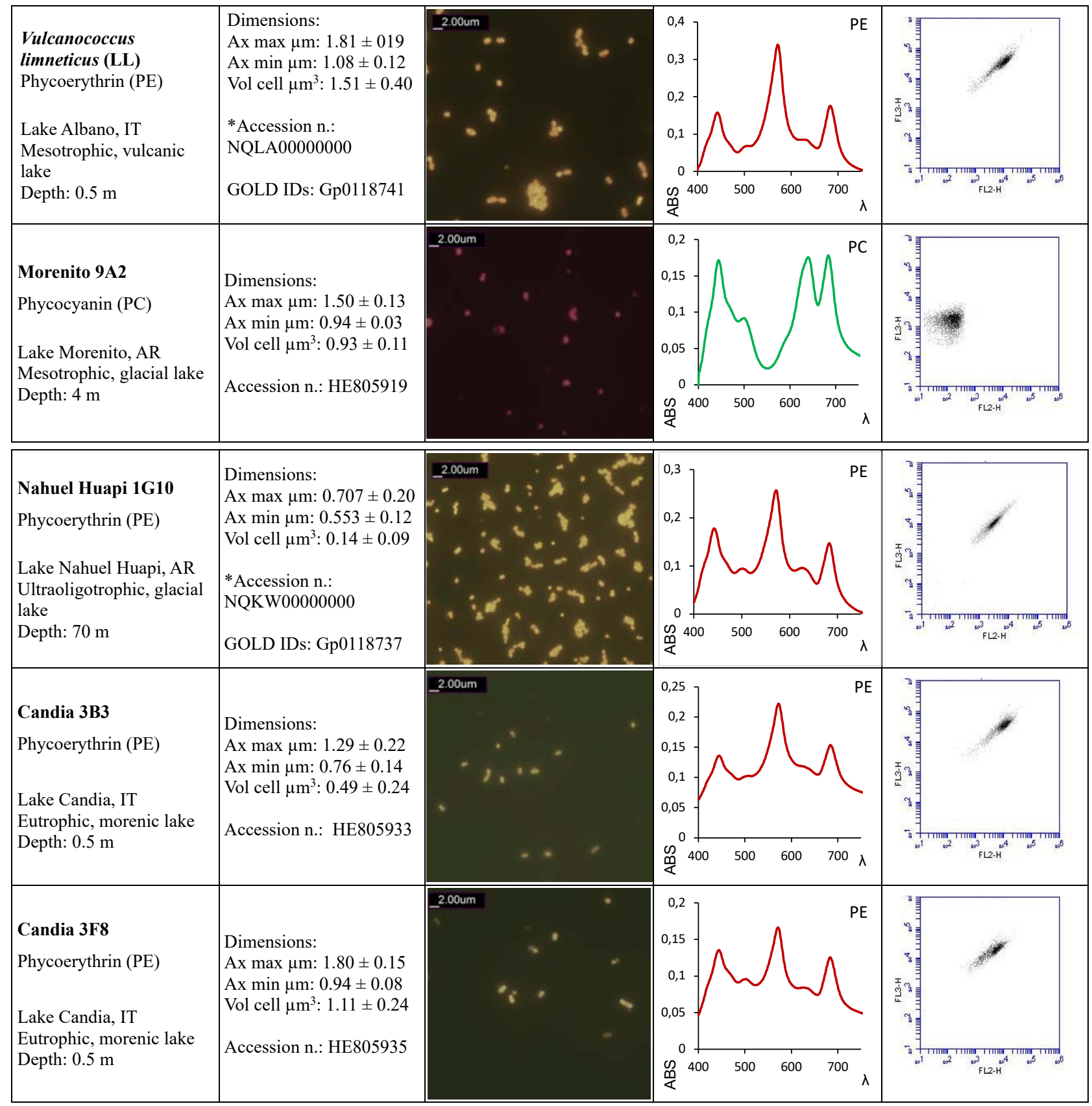

To be continued on next page 
Table 1. Continued from previous page.

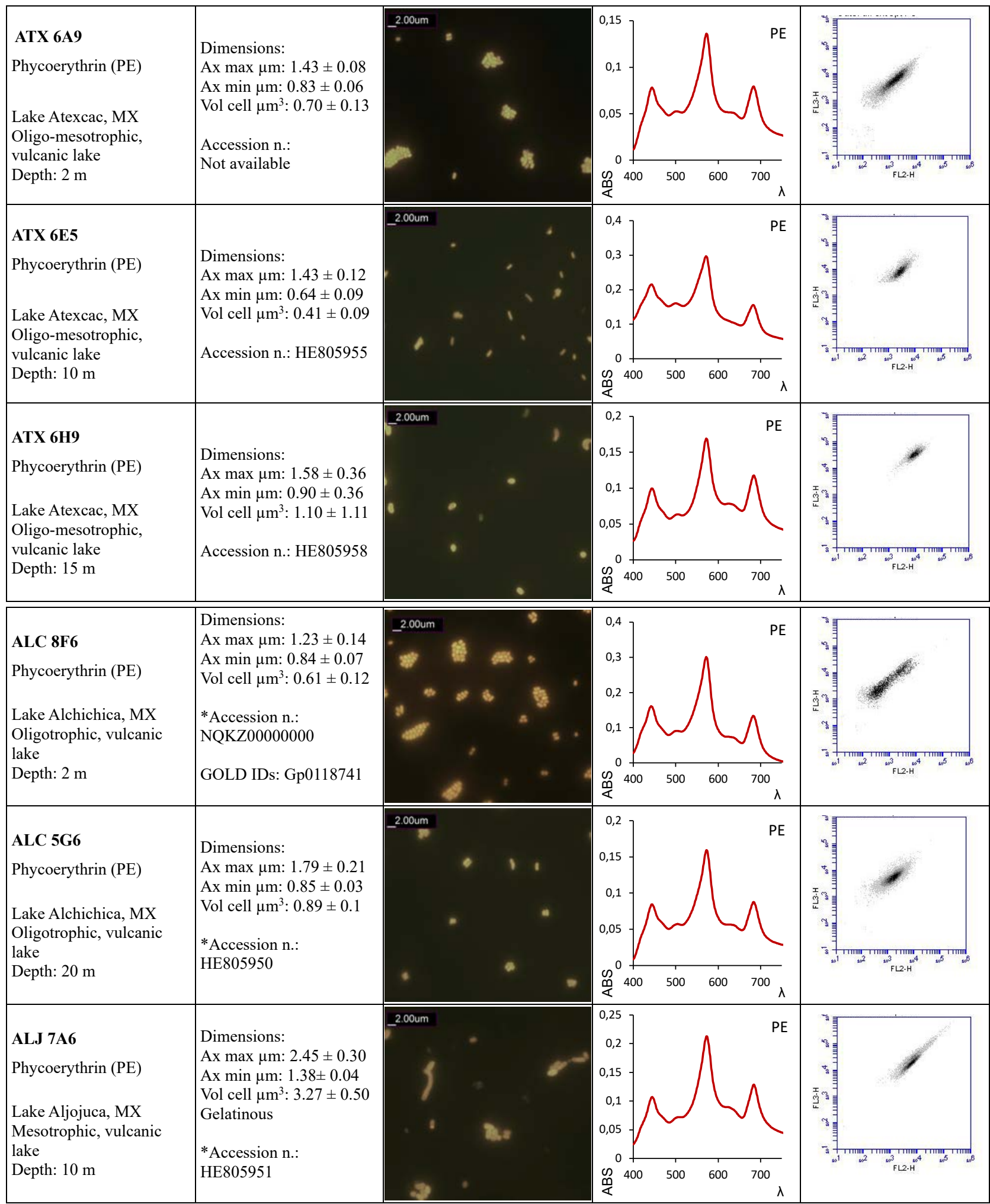


Table 1. Continued from previous page.

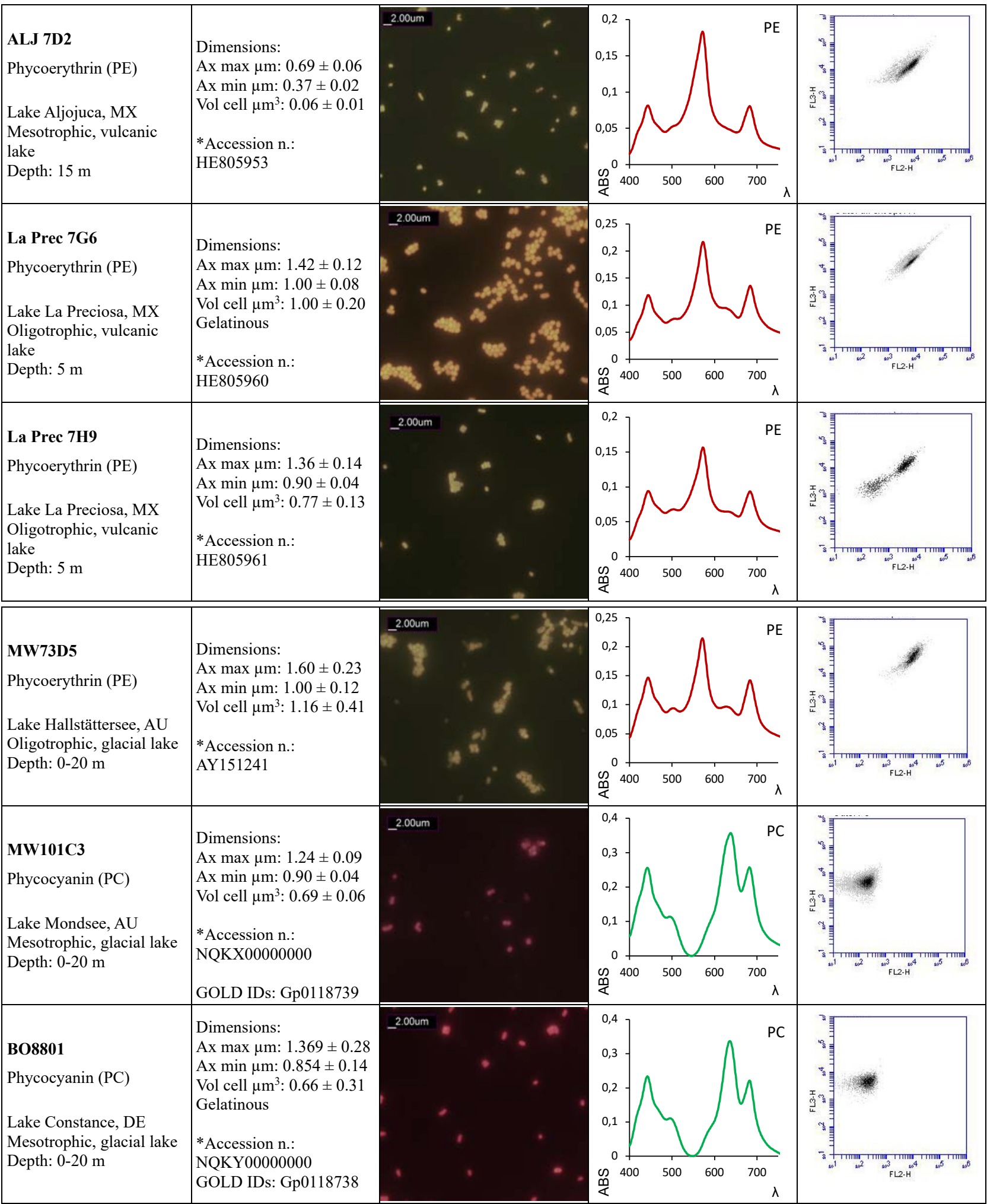


Table 1. Continued from previous page.

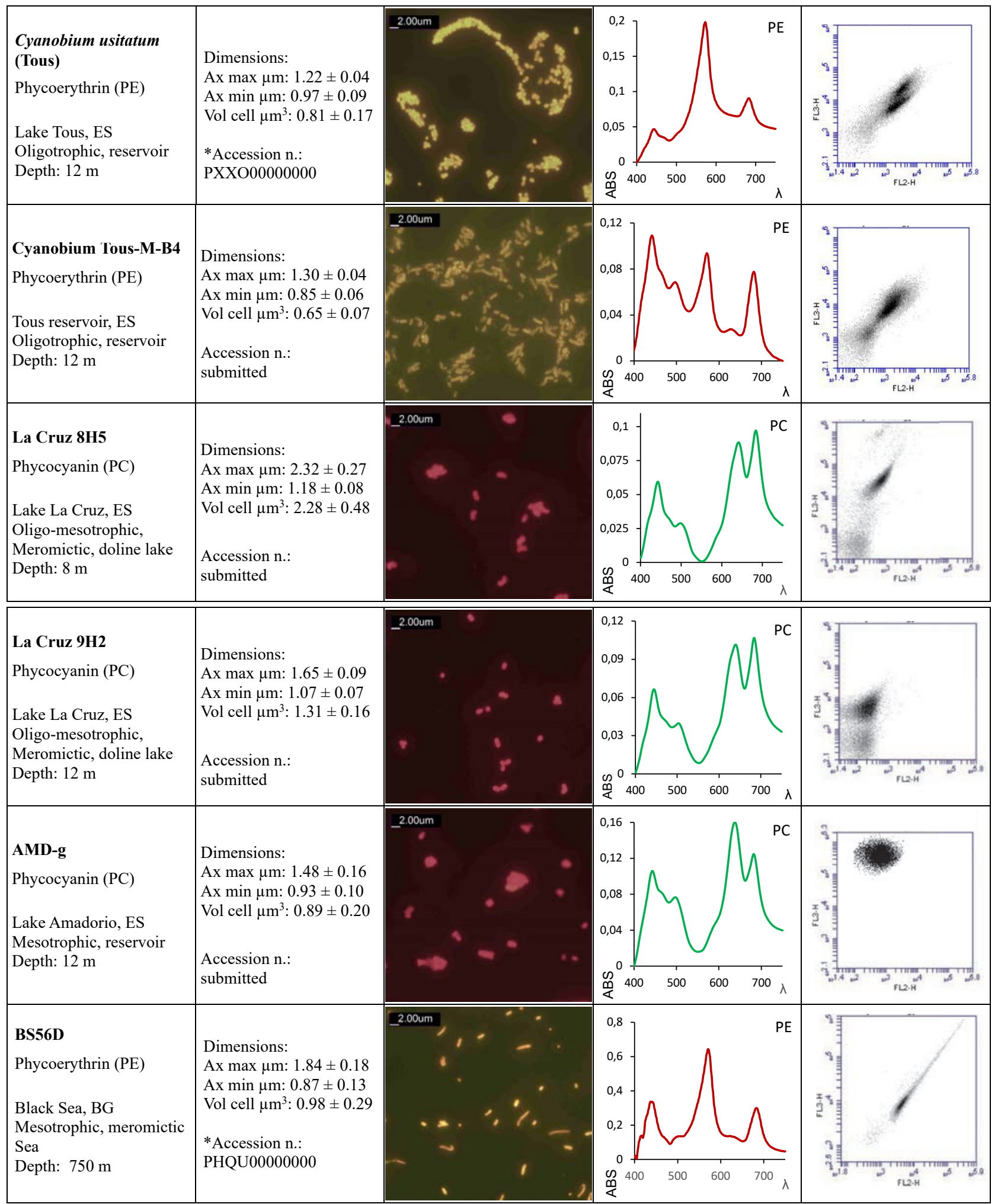


Table 1. Continued from previous page.

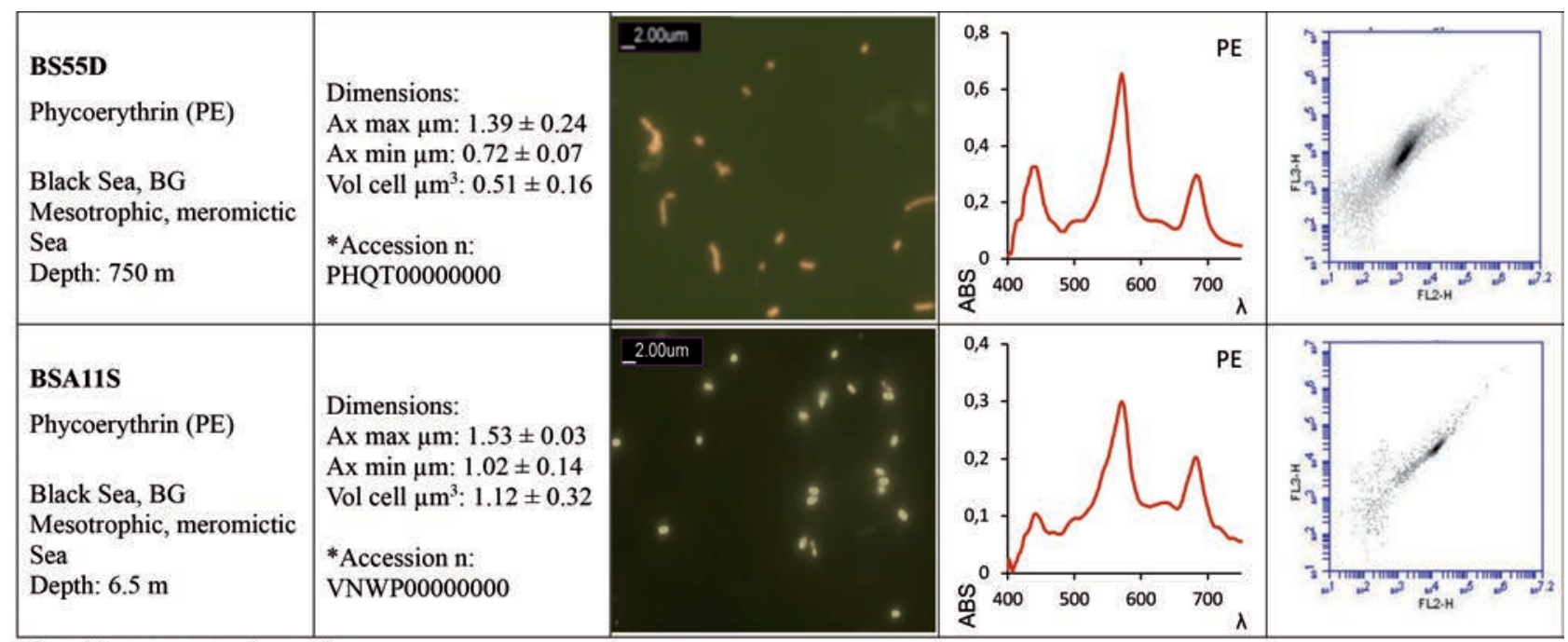

*Complete genome accession number.

excitation to the reaction centre.

The twenty-five picocyanobacterial strains that were selected from the CNR-IRSA collection represent: i) seven different 16S rRNA gene phylogenetic groups (group A, $C$. gracile; group B, Subalpine cluster I; undefined group, L. Atexcac Mexico; unnamed group; Halotolerants group; Nahuel Huapi group; group I) (Callieri et al., 2013); and ii) eleven different phylogenomic groups, based on a new phylogenomic tree (Fig. 2). The tree contains previously studied freshwater representatives (Cabello-Yeves et al., 2018; Sanchez-Baracaldo et al., 2019) and other sequenced representatives from all known sub-clusters and clades. The majority of our strains are affiliated with the polyphyletic sub-cluster 5.2, which comprises brackish, estuarine, euryhaline and freshwater species. Candia 3F8, 3B3 and MW73D5 are strains with $>99 \%$ of Average Nucleotide Identity (ANI) to Vulcanococcus limneticus LL (Di Cesare et al., 2018). Initially, these strains were named as Synechococcus, and were already published in a previous 16S rRNA tree under this nomenclature (Callieri et al., 2013). Recently, we opened up the possibility for a new genus Vulcanococcus and species limneticus naming only for taxonomic purposes, but we acknowledge the use of both nomenclatures. There are also two marine strains coming from the Black Sea $(750 \mathrm{~m})$ that are affiliated to the sub-cluster $5.1 \mathrm{~A} / \mathrm{B}$. These isolates are positioned near halotolerant strains WH8101 (Atlantic Ocean) and RS9916 (Red Sea) (Fuller et al. 2003, Doré et al., 2020)

\section{MATERIALS AND METHODS}

\section{Isolation and culture media}

A detailed description of sampling type, sampling collection, isolation and purification can be found in
Callieri et al., (2013), and Cabello-Yeves et al., (2018). Briefly, water samples were filtered through a $3 \mu \mathrm{m}$ polycarbonate membrane directly after sampling, on boat or at lake shore (or in the laboratory if nearby). Then, the filtrate water was added into a sterile tube containing BG11 medium and kept in fresh and low light container until arrival to the laboratory, where they were cultured on a climate chamber at $18-20^{\circ} \mathrm{C}$ under low light intensity (10$15 \mu \mathrm{mol}$ photons $\left.\mathrm{m}^{-2} \mathrm{~s}^{-1}\right)$. Finally, strain isolation was performed with flow cytometric single-cell sorting (InFlux V-GS flow cytometer, Becton Dickinson Inc.). For a more detailed description on the entire procedure and the culture media preparation, see supplementary materials.

\section{Microscopy, cytometry and spectrophotometry}

The morphology of the different picocyanobacteria strains was observed by epifluorescence microscope. Briefly, appropriate cell dilutions from cultures growing at $20^{\circ} \mathrm{C}$ with light intensity of $10 \mu \mathrm{mol} \mathrm{m} \mathrm{s}^{-1}$ were collected on $0.2 \mu \mathrm{m}$ white polycarbonate filters (Nucleopore). The filters were mounted on microscope slides with antirefractive immersion oil (Cargille Type FF) and visualized by epifluorescence microscope (Axioplan, ZEISS) using filter sets for blue (BP450-490, FT510, LP520) and green light excitation (LP510-560, FT580, LP590), adopted for the detection of phycoerythrin (PE) and phycocyanin (PC) autofluorescence, respectively (Callieri et al., 2011). For the cell measurements, a minimum of 100 cells were counted for each strain, in at least 10 different fields at a magnification of 1250x. The final average cell dimension was calculated with the help of a semi-automated image software (Optimas) that elaborated the images taken by a highly sensible camera (Silicon intensified Target TV) equipped to the microscope. 
All cultures were further analysed using a flow cytometer (Accuri C6, Becton Dickinson, HJ, USA) equipped with a $20 \mathrm{~mW} 488 \mathrm{~nm}$ solid state blue laser and a $14.7 \mathrm{~mW} 640 \mathrm{~nm}$ diode red laser. The forward (FSC) and side light scattering (SSC) signals from green $(\mathrm{FL} 1$ channel $=533 / 30 \mathrm{~nm})$, orange $(\mathrm{FL} 2$ channel $=$ $585 / 40 \mathrm{~nm}$ ) and red fluorescence (FL3 channel $>670 \mathrm{~nm}$ and FL4 channel 675/25) were acquired and considered for the characterization of the different strains. Cells were characterized and distinguished according to their distinct pigment composition (i.e., reflecting on different intensities of autofluorescence signals collected at the orange and red channels, Croce and van Amerongen, 2014) and size (i.e., proportionally related to light scatter signals, Tzur et al. 2011) using a semi-automated software (BD AccuritM C6) installed in the flow cytometer. With threshold values set at 1000 for the FL3 channel and at 2000 for the FL4 channel (or 7000 for FSC-H and 1500 for FL4-H) the density plots of FL2-H vs FL3-H and FL3-H vs FL4-H allowed for the optimal gating design and quantification.

Absorbance spectra (450-750nm) of the strains were obtained using a UV-mc2 spectrophotometer (Safas, Monaco). Samples were adequately diluted in a $1 \mathrm{~cm}$ quartz cuvette and analyzed before and after the addition of $50 \mu \mathrm{l} \mathrm{NaClO}$ (for removal of pigments, Ferrari and Tassan, 1999). The absorbance spectra with minimized scattering contribute were obtained by subtracting the spectrum recorded after the $\mathrm{NaClO}$ treatment to the one of the untreated diluted sample (Kishino et al., 1985).
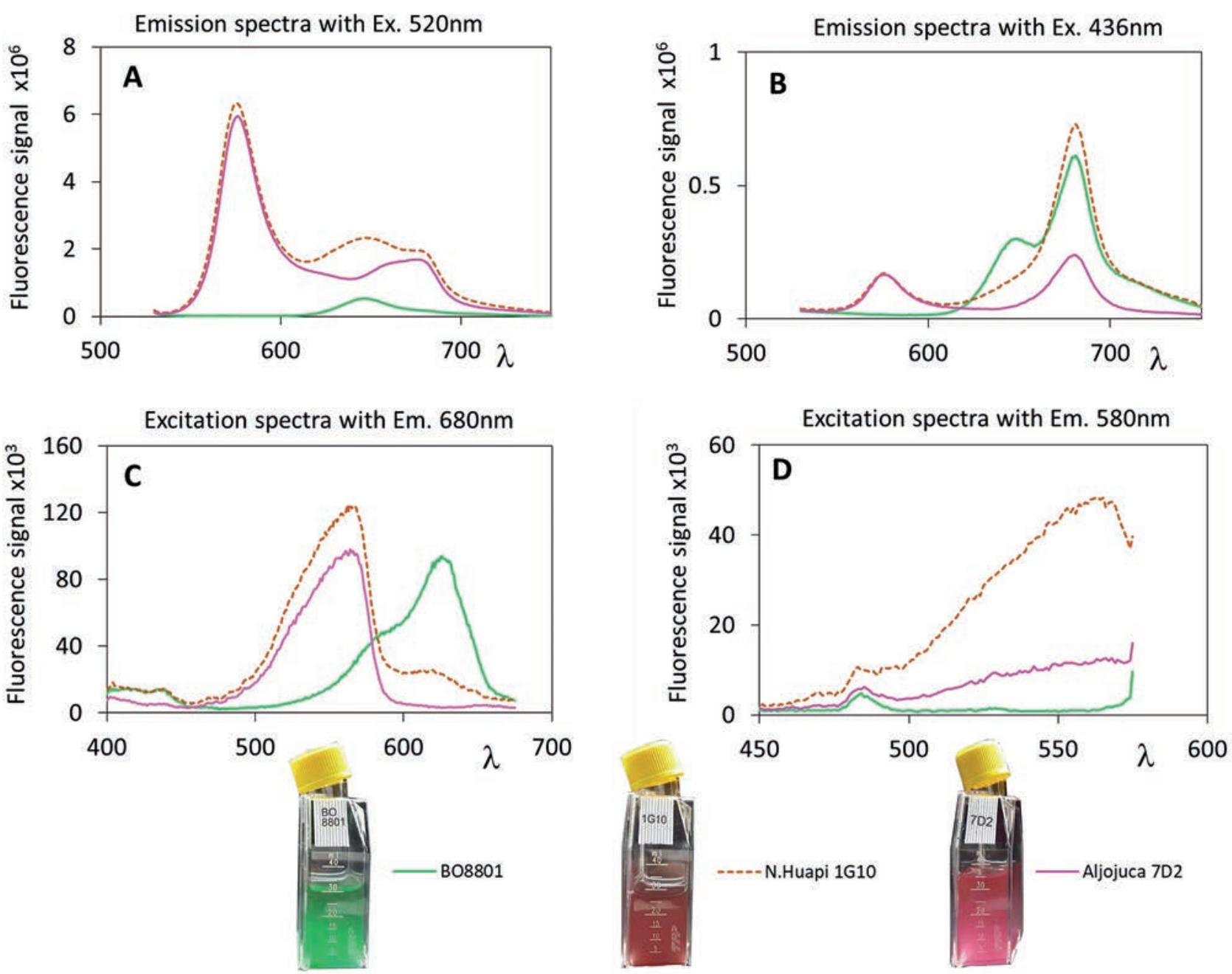

Fig. 1. Emission and excitation spectra of three Synechococcus strains with different accessory pigments BO8801 (PC, green), Nahuel Huapi 1 G10 (PE, orange) and Aljojuca 7D2 (PE, pink). Emission spectra with excitation at $520 \mathrm{~nm}$ (A) and $436 \mathrm{~nm}$ (B). Excitation spectra with emission at $680 \mathrm{~nm}$ (C) and $580 \mathrm{~nm}$ (D). The native cultures for each strain is also included, displaying the typical colour given by their unique pigmentation. 


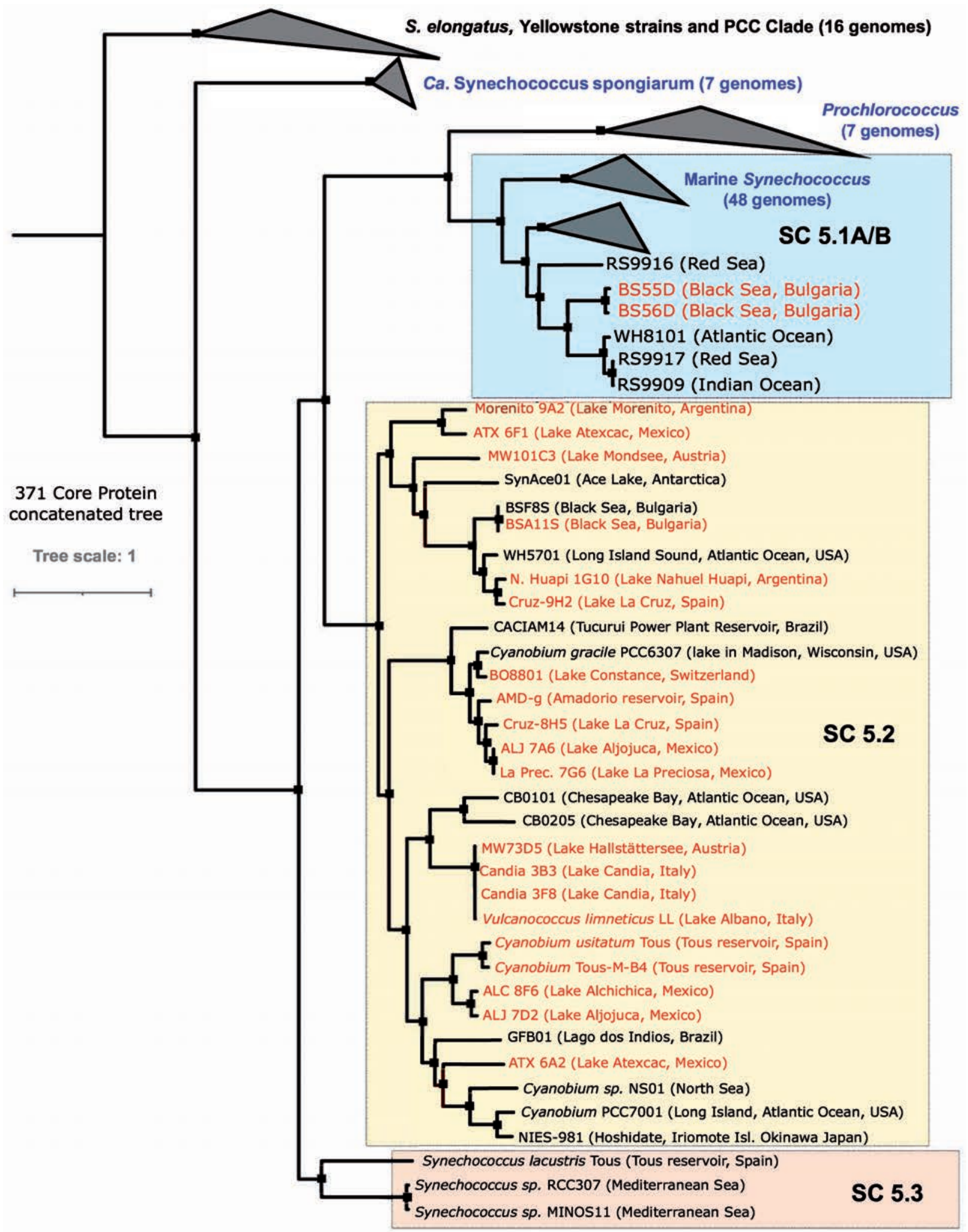

Fig. 2. Protein-concatenated phylogenomic tree constructed with 371 core proteins with PhyloPhlAn tool. The tree includes all culturederived picocyanobacteria from Synechococcus and Cyanobium genera inside sub-clusters 5.1, 5.2 and 5.3, a few Prochlorococcus representatives and Ca. Synechococcus spongiarum. The tree was rooted at the S. elongatus and PCC clade. All sequenced cultures derived from this collection and included in Tab. 1 are red coloured. Bootstrap values higher than 0.95 are marked as black squares on nodes. The scale bar represents the number of mutations/site. 


\section{Spectrofluorimetric analysis}

Emission and excitation spectra for selected strains, BO8801 (PC), Nahuel Huapi 1G10 (PE) and Aljojuca 7D2 (PE), were carried out with a FluoroMax-4 spectrofluorometer (HORIBA Scientific, Irvine, CA, USA) to well characterize their representative content in phycobiliproteins, highly fluorescent molecules.

Excitation spectra were executed setting the emission wavelength $\left(\lambda_{\mathrm{em}}\right)$ at $680 \mathrm{~nm}$ (chlorophyll- $a$ emission peak) and $580 \mathrm{~nm}$ (phycoerythrin emission peak). On the other hand, emission spectra were executed at an excitation wavelength $\left(\lambda_{\mathrm{ex}}\right)$ of $436 \mathrm{~nm}$ (one of the chlorophyll- $a$ absorbance peak) and $520 \mathrm{~nm}$ (shorter wavelength than the phycoerythrin absorbance peak). Excitation spectra at $\lambda_{\mathrm{em}}=680$ and $580 \mathrm{~nm}$ and emission spectra at $\lambda_{\mathrm{ex}}=436$ and $520 \mathrm{~nm}$ were chosen to compare them with already published works that evaluate the colour differences between PE cells and PC cells (Callieri et al., 1996). The fluorescence spectra of the cultures were measured in a 1 $\mathrm{cm}$ quartz cuvette, with an excitation and emission slits of $10 \mathrm{~nm}$ and scan speed of $1 \mathrm{~nm} \mathrm{~s}^{-1}$. Samples' spectra were corrected by subtracting the spectrum of the medium BG-11 (blank).

\section{DNA extraction, sequencing and phylogenomics}

DNA extraction, sequencing and phylogenomic analyses of the strains were already described in detail in previous works (Cabello-Yeves et al., 2018; Di Cesare et al., 2018; Sanchez-Baracaldo et al., 2019). Briefly, $2 \mathrm{ml}$ of highly concentrated culture, in order to obtain a sufficient pellet to extract DNA, were centrifuged. DNA was extracted from medium-free pellets using the UltraClean Microbial DNA Isolation kit (MOBio Laboratories, Inc., Carlsbad, CA, USA) according to manufacturer's instruction. E.Z.N.A. ${ }^{\circledR}$ Soil DNA Kit (Omega Bio-tek) and phenol-chloroform DNA extraction methodologies were also applied for some strains (Cabello-Yeves et al., 2018). Strains were sequenced with Illumina HiSeq or MiSeq and draft genomes were obtained from an assembly step with SPAdes assembler following 'auto' parameters (Bankevich et al., 2012). A 371 protein-concatenated phylogenomic tree was constructed using the tool PhyloPhlAn (Segata et al., 2013), which comprised all culture-derived picocyanobacteria from Synechococcus and Cyanobium genera inside sub-clusters 5.1, 5.2 and 5.3. A FastTree with 100 bootstraps was constructed with the above mentioned tool. For the construction of the tree, no sequences were available of strains ATX 6A9 and ATX $6 \mathrm{H} 9$. Instead, they were represented in the phylogenomics by their closely related picocyanobacteria ATX 6A2 and ATX 6F1, respectively. The universal markers of strains Cyanobium Tous-M-B4, La Cruz 8H5, La Cruz 9H2 and AMD-g were gently granted and used for the phylogenomic tree, but were not yet published in databases by the time this atlas was written.

\section{REFERENCES}

Bankevich A, Nurk S, Antipov D, Gurevich AA, Dvorkin M, Kulikov AS, et al., 2012. SPAdes: a new genome assembly algorithm and its applications to single-cell sequencing. J. Comput. Biol. 19:455-477.

Cabello-Yeves PJ, Haro-Moreno JM, Martin-Cuadrado AB, Ghai R, Picazo A, Camacho A, Rodriguez-Valera F, 2017. Novel Synechococcus genomes reconstructed from freshwater reservoirs. Front. Microbiol. 8:1151.

Cabello-Yeves PJ, Picazo A, Camacho A, Callieri C, Rosselli R, Roda-Garcia JJ, et al., 2018. Ecological and genomic features of two widespread freshwater picocyanobacteria. Environ. Microbiol. 20:3757-3771.

Callieri C, Amicucci E, Bertoni R, 1996. Fluorometric characterization of two picocyanobacteria strains from lakes of different underwater light quality. Int. Revue Ges. Hydrobiol. 81:13-23.

Callieri C, Lami A, Bertoni R, 2011. Microcolony formation by single-cell Synechococcus strains as a fast response to UV radiation. Appl. Environ. Microbiol. 77:7533-7540.

Callieri C, Cronberg G, Stockner J, 2012. Freshwater picocyanobacteria: single cells, microcolonies and colonial forms, p. 229-269. In: B. Whitton (ed.), Ecology of Cyanobacteria: Their Diversity in Time and Space. Springer.

Callieri C, Coci M, Corno G, Macek M, Modenutti B, Balseiro E, Bertoni R, 2013. Phylogenetic diversity of nonmarine picocyanobacteria. FEMS Microbiol. Ecol. 85:293-301.

Callieri C, Slabakova V, Dzhembekova N, Slabakova N, Peneva E, Cabello-Yeves PJ. et al., 2019. The mesopelagic anoxic Black Sea as an unexpected habitat for Synechococcus challenges our understanding of global "deep red fluorescence". ISME J. 13(7):1676-1687

Croce R, Van Amerongen H, 2014. Natural strategies for photosynthetic light harvesting. Nature Chem. Biol. 10:492-501.

Di Cesare A, Cabello-Yeves PJ, Chrismas NAM, SánchezBaracaldo P, Salcher MM, Callieri C, 2018. Genome analysis of the freshwater planktonic Vulcanococcus limneticus sp. nov. reveals horizontal transfer of nitrogenase operon and alternative pathways of nitrogen utilization. BMC Genomics. 19:259

Doré H, Farrant GK, Guyet U, Haguait J, Humily F, Ratin M, et al., 2020. Evolutionary mechanisms of long-term genome diversification associated with niche partitioning in marine picocyanobacteria. Front. Microbiol. 11:567431.

Dufresne A, Ostrowski M, Scanlan DJ, Garczarek L, Mazard S, Palenik BP, et al., 2008. Unraveling the genomic mosaic of a ubiquitous genus of marine cyanobacteria. Genome Biol 9: R90.

Farrant GK, Dore H, Cornejo-Castillo FM, Partensky F, Ratin M, Ostrowski M, et al., 2016. Delineating ecologically significant taxonomic units from global patterns of marine picocyanobacteria. Proc. Natl. Acad. Sci. U S A 113:E3365E3374.

Ferrari GM, Tassan S, 1999. A method using chemical oxidation 
to remove light absorption by phytoplankton pigments. J. Phycol. 35:1090-1098.

Flombaum P, Gallegos JL, Gordillo A, Rincon J, Zabala LL, Jiao N, et al., 2013. Present and future global distributions of the marine Cyanobacteria Prochlorococcus and Synechococcus. Proc. Natl. Acad. Sci. USA 110:9824-9829.

Fuller NJ, Marie D, Partensky F, Vaulot D, Post AF, Scanlan D, 2003. Clade-specific 16S Ribosomal DNA oligonicleotides reveal the predominance of a single Synechoocccus clade throughout a stratified water column in the red sea. Appl. Environ. Microbiol. 69:2430-2443.

Hamilton TL, Bryant DA, Macalady JL, 2016. The role of biology in planetary evolution: cyanobacterial primary production in low oxygen Proterozoic oceans. Environ. Microbiol. 18:325-340.

Kishino M, Takahashi M, Okami N, Ichimura S, 1985. Estimation of the spectral absorption coefficients of phytoplankton in the sea. Bull. Mar. Sci. 37:634-642.

Olson RJ, Zettler ER, Anderson OK, 1989. Discrimination of eukaryotic phytoplankton cell types from light scatter and autofluorescence properties measured by flow cytometry. Cytometry. 10:636-643.

Ong LJ, Glazer AN, 1991. Phycoerythrins of marine unicellular cyanobacteria .3. Sequence of a Class-II Phycoerythrin. J. Biological Chem. 266:9535-9539.

Sanchez-Baracaldo P, Bianchini G, Di Cesare A, Callieri C, Chrismas NA, 2019. Insights into the evolution of picocyanobacteria and phycoerythrin genes (mpeBA and cpeBA). Front. Microbiol 10:45.

Scanlan DJ, Ostrowski M, Mazard S, Dufresne A, Garczarek L, Hess WR, et al., 2009. Ecological genomics of marine picocyanobacteria. Microbiol. Mol. Biol. Rev. 73: 249-299.

Scanlan D, 2012. Marine Picocyanobacteria, p. 503-533. In: B. Whitton (ed.), Ecology of Cyanobacteria: Their Diversity in Time and Space. Springer.

Segata N, Börnigen D, Morgan XC, Huttenhower C, 2013. PhyloPhlAn is a new method for improved phylogenetic and taxonomic placement of microbes. Nature Comm. 4:1-11.

Six C, Thomas JC, Garczarek L, Ostrowski M, Dufresne A, Blot N, et al., 2007. Diversity and evolution of phycobilisomes in marine Synechococcus spp.: a comparative genomics study. Genome Biol. 8:R259.

Stomp M, Huisman J, Vörös L, Pick FR, Laamanen M, Haverkamp T, Stal LJ, 2007. Colorful coexistence of red and green picocyanobacteria in lakes and seas. Ecol. Lett. 10:290-298.

Tzur A, Moore JK, Jorgensen P, Shapiro HM, Kirschner MW, 2011. Optimizing optical flow cytometry for cell volumebased sorting and analysis. PloS One 6:e16053.

Wood MA, Horan PK, Muirhead K, et al., 1985. Discrimination between types of pigments in marine Synechococcus spp. by scanning spectroscopy, epifluorescence microscopy, and flow cytometry. Limnol. Oceanogr. 30:1303-1315. 